\title{
BIBLIOGRAPHY
}

1. S. C. Kleene, Introduction to metamathematics, New York, Amsterdam, and Groningen, 1952.

2. R. M. Robinson, Review, J. Symbolic Logic vol. 16 (1951) p. 282.

3. P. C. Rosenbloom, An elementary constructive proof of the fundamental theorem of algebra, Amer. Math. Monthly vol. 52 (1945) pp. 562-570.

4. E. Specker, Nicht Konstructiv beweisbare Sätze der Analysis, J. Symbolic Logic vol. 14 (1949) pp. 145-158.

5. A. M. Turing, On computable numbers, with an application to the Entscheidungsproblem, Proc. London Math. Soc. (2) vol. 42 (1936-37) pp. 230-265.

UNIVERSITY OF NEW HAMPSHIRE

\section{A THEOREM ON HYPERSIMPLE SETS}

\section{J. C. E. DEKKER}

Introduction. E. L. Post proved in his paper Recursively enumerable sets of positive integers and their decision problems ${ }^{1}$ that a creative set cannot be reducible to a hypersimple set by truth tables (pp. 308310 ). The present paper is concerned with the question whether a creative set can be Turing reducible to a hypersimple set. It will be shown that the answer to this question is affirmative. In fact, we shall establish the following more general statement: for every recursively enumerable, but not recursive, set $\alpha$, a hypersimple set $\beta$ can be effectively found such that $\alpha$ and $\beta$ are Turing reducible to each other.

Preliminaries. A non-negative integer is called a number, a collection of numbers is called a set and a collection of sets a class. A set is immune, if it is infinite, but has no infinite recursively enumerable subset. For the definitions of a discrete array and a hypersimple set we refer to our paper Two notes on recursively enumerable sets ${ }^{2}$ (p. 497). We shall use the following notations: " $\rho(f)$ " for the range of the function $f(n)$, " $E$ " for the class of all recursive sets, " $F$ " for the class of all recursively enumerable (r.e.) sets, " $\alpha t$-red $\beta$ " for $\alpha$ is reducible to $\beta$ by truth tables, and " $\alpha$ red $\beta$ " for $\alpha$ is Turing reducible to $\beta$. If $a\left(t_{1}\right)$ and $a\left(t_{2}\right)$ are elements of the sequence $\{a(n)\}$, we say that $a\left(t_{2}\right)$ succeeds $a\left(t_{1}\right)$ in $\{a(n)\}$ if $t_{2}>t_{1}$.

Definition. Let $a(n)$ be a 1-1 function. The element $a(t)$ of the \footnotetext{
1953.

Presented to the Society, November 27, 1953; received by the editors November 5 ,

1 Bull. Amer. Math. Soc. vol. 50 (1944) pp. 284-316.

2 Proc. Amer. Math. Soc. vol. 4 (1953) pp. 495-501.
} 
sequence $\{a(n)\}$ is called minimal if $a(t)<a(t+k)$ for every $k \geqq 1$. The set of all elements in $\{a(n)\}$ which are not minimal is denoted by "hyp $a(n)$."

Definition. The subset $\sigma$ of $\alpha$ is called hypersimple relative to $\alpha$ if $\sigma$ is r.e., $\alpha-\sigma$ infinite, and $\sigma$ includes at least one row of every discrete array all of whose elements belong to $\alpha$.

Let $a(n)$ be a 1-1 recursive function. It is now readily verified that the subset $\sigma$ of $\rho(a)$ is hypersimple relative to $\rho(a)$ if and only if $a^{-1}(\sigma)$ is hypersimple.

\section{The main result.}

TheOREM 1. For every set $\alpha \in F-E$ one can effectively find a r.e. set $\beta$ such that: (1) $\beta$ is hypersimple, (2) $\beta$-red $\alpha$, (3) $\alpha \operatorname{red} \beta$.

Proof. Let $a(n)$ be a 1-1 recursive function ranging over $\alpha, \zeta$ $={ }_{d f}$ hyp $a(n)$ and $\beta={ }_{d j} a^{-1}(\zeta)$. We claim that $\beta$ satisfies the requirements.

(1) It suffices to show that $\zeta$ is hypersimple relative to $\alpha$. We shall call an element of $\alpha$ minimal if it is minimal relative to $\{a(n)\}$. For every number $k$ we can effectively list the elements in $a(0), \cdots$, $a(k)$ which are succeeded by a smaller element in this finite sequence. Since we can do this for $k=1,2, \cdots$, we conclude that $\zeta$ is r.e. Suppose $a\left(k_{0}\right)$ is the smallest element of $\alpha$, then $a\left(k_{0}\right)$ is minimal; if $a\left(k_{1}\right)$ is the smallest element of $\alpha-\left\{a(0), \cdots, a\left(k_{0}\right)\right\}, a\left(k_{1}\right)$ is also minimal and $a\left(k_{0}\right)<a\left(k_{1}\right)$. Continuing this procedure we see that $\alpha$ contains infinitely many minimal elements, i.e., that $\alpha-\zeta$ is infinite. Let $\left\{\delta_{n}\right\}$ be a discrete array all of whose elements belong to $\alpha$. Then there exist recursive functions $d(n, x)$ and $e(n)$ such that for every $n, \delta_{n}=\{d(n, 0), \cdots, d(n, e(n))\}$; we may assume without loss of generality that $d(n, x)$ is strictly increasing for every $n$. We know that $\alpha$ is not recursive. To prove that $\zeta$ includes at least one row of $\left\{\delta_{n}\right\}$ it suffices therefore to show that the assumption that $\zeta$ includes no row of $\left\{\delta_{n}\right\}$ implies that $\alpha$ is recursive. This can be done in the following manner. Suppose $\zeta$ included no row of $\left\{\delta_{n}\right\}$. Then every row of $\left\{\delta_{n}\right\}$ would contain at least one minimal element. Observe that $d(n, 0)$ is a $1-1$ recursive function of $n$ such that $d(n, 0)=\min \delta_{n}$ for every $n$. Let $x$ be any number. Find the first element in $\{d(n, 0)\}$ which is greater than $x$, say $d(r, 0)$. Then $\delta_{r}$ contains a minimal element, say $d(r, s)$. Thus $x<d(r, 0) \leqq d(r, s) \leqq d(r, e(r))$. We can now locate the elements of $\delta_{r}$ in $\{a(n)\}$, i.e., we can effectively find the numbers $n_{0}, \cdots, n_{e(r)}$ such that $d(r, 0)=a\left(n_{0}\right), \cdots, d(r, e(r))$ $=a\left(n_{e(r)}\right)$. Let $t_{x}={ }_{d f} \max \left(n_{0}, \cdots, n_{e(r)}\right)$. The element $a\left(t_{x}\right)$ is now 
either equal to the minimal element $d(r, s)$ or it succeeds $d(r, s)$ in $\{a(n)\}$. It now follows from the fact that $d(r, s)>x$ that all elements in $\{a(n)\}$ which succeed $a\left(t_{x}\right)$ in $\{a(n)\}$ are greater than $x$. Hence $x \in \alpha$ if and only if $x \in\left\{a(0), \cdots, a\left(t_{x}\right)\right\}$. Since $t_{x}$ is a recursive function of $x$, we would now possess an effective procedure to test whether $x$ belongs to $\alpha$. Thus $\alpha$ would be recursive.

(2) Let $x$ be any number. Then $x \in \beta$ if and only if $a(x)$ is not minimal. Let $\alpha_{x}^{*}={ }_{d f}\{0, \cdots, a(x)-1\}-\{a(0), \cdots, a(x-1)\}$, then $a(x)$ is not minimal if and only if $\alpha_{x}^{*}$ contains at least one element of $\alpha$. There now exist recursive functions $a_{x, n}^{*}$ and $m(x)$ such that:

(a) if $\alpha_{x}^{*}$ is empty, then $a_{x, 0}^{*}=0$ and $m(x)=0$,

(b) if $\alpha_{x}^{*}$ is nonempty, then

$$
\alpha_{x}^{*}=\left\{a_{x, 0}^{*}, \cdots, a_{x, m(x)}^{*}\right\} .
$$

Let $T_{x}$ be the truth table of order $m(x)+1$ whose last column is - , in case $\alpha_{x}^{*}$ is empty and is $+,+, \cdots,+,-$ in case $\alpha_{x}^{*}$ is nonempty. The decision problem of $\beta$ is now reducible to that of $\alpha$ by the reduction which associates with every number $x$ the finite sequence $a_{x, 0}^{*}, \cdots, a_{x, m(x)}^{*}$ and the truth table $T_{x}$. Thus $\beta t$-red $\alpha$.

(3) Let $x$ be any number. We can effectively find a recursive function $h_{n}$ (depending on $x$ ) such that all elements of $\alpha$ which are greater than $x$ are generated in the sequence $\left\{a\left(h_{n}\right)\right\}$. We now raise the following questions about $\zeta$ :

$$
\text { " } a\left(h_{0}\right) \in \zeta ?, " \quad \text { " } a\left(h_{1}\right) \in \zeta ?, " \cdots .
$$

After a finite number of questions the correct answer must be negative, since $\alpha-\zeta$ is infinite and all but finitely many elements of $\alpha$ are assumed by $a\left(h_{n}\right)$. Let $p(x)$ be the first $n$ such that $a\left(h_{n}\right) \notin \zeta$. Then we know for every $x$ that $a\left(h_{p(x)}\right)$ is a minimal element of $\alpha$ greater than $x$. Thus $x \in \alpha$ if and only if $x \in\left\{a(0), \cdots, a\left(h_{p(x)}-1\right)\right\}$. Let $c_{\alpha}(x)$, $c_{\beta}(x), c_{\zeta}(x)$ be the characteristic functions of $\alpha, \beta, \zeta$ respectively. We have proved that $c_{\alpha}(x)$ is recursive in $c_{\zeta}(a(x))$. But $c_{\zeta}(a(x))=c_{\beta}(x)$. It follows that $c_{\alpha}(x)$ is recursive in $c_{\beta}(x)$, i.e., that $\alpha$ red $\beta$.

REMARK 1. In the proof of (1) no use is made of the fact that there exists a hypersimple set. As a side result we have therefore a new existence proof for hypersimple sets.

REMARK 2. While the fact that $\alpha=\rho(a)$ is not recursive plays an essential role in the proof of (1), it is not used in the proof of (2). The statement $\beta t$-red $\alpha$ is therefore still valid if $\alpha$ is recursive. Thus, if $\alpha$ is recursive, $\beta$ is recursive. This can also be seen in the following way. If $a(n)$ is a 1-1 recursive function, ranging over the recursive set $\alpha$, 
we can decide for any $n$ whether $a(n)$ is minimal in $\{a(n)\}$ by locating $a(n)$ in the recursive enumeration of $\alpha$ according to size.

Corollary 1. There exist two r.e. sets $\sigma$ and $\tau$ such that $\sigma t$-red $\tau$ is false, but $\sigma$ red $\tau$ true.

Proof. Let $\sigma$ be any creative set, $s(n)$ a 1-1 recursive function ranging over $\sigma$, and $\tau={ }_{d f} s^{-1}$ (hyp $s(n)$ ). Then $\sigma$ red $\tau$ is true; but $\sigma t$-red $\tau$ is false, because a creative set cannot be reducible to a hypersimple set by truth tables.

COROLlary 2. There exist two sets in $F-E$ which are not Turing reducible to each other if and only if there exist two hypersimple sets which are not Turing reducible to each other.

\section{A generalization.}

Definition. Let $a(n)$ be a $1-1$ function and $t$ any number. Then the deficiency dfc $a(t)$ of the element $a(t)$ in $\{a(n)\}$ is the number of elements which while smaller than $a(t)$ succeed $a(t)$ in $\{a(n)\}$.

Let $a(n)$ be a 1-1 recursive function. It is now easily verified that the function $\operatorname{dfc} a(n)$ is recursive if and only if the set $\rho(a)$ is recursive. For let $\rho(a)$ be recursive. Then the statements

$$
0 \in \rho(a), 1 \in \rho(a), \cdots, a(n)-1 \in \rho(a)
$$

can be effectively tested for every $n$; the results can then be compared with the elements $a(0), \cdots, a(n-1)$. Thus dfc $a(n)$ is recursive. To establish the converse, suppose $\rho(a)$ is not recursive. Then the set $\lambda$ of all elements in $\{a(n)\}$ which have deficiency 0 (i.e., which are minimal) is immune by Theorem 1 . But, if $\operatorname{dfc} a(n)$ were recursive, $\lambda$ would be r.e. We conclude that $\operatorname{dfc} a(n)$ is not recursive.

THEOREM 2. Let $a(n)$ be a 1-1 recursive function such that $\alpha=\rho(a)$ $\in F-E$. Let $k$ be any number $\geqq 1$ and $\beta_{k}={ }_{d f}\{n \mid \operatorname{dfc} a(n) \geqq k\}$. Then we know: (1) $\beta_{k}$ is hypersimple, (2) $\beta_{k} t$-red $\alpha$, (3) $\alpha \operatorname{red} \beta_{k}$.

Proof. Let $\zeta_{k}={ }_{d f} a\left(\beta_{k}\right)$. It is now easily verified that $\zeta_{k}$ is r.e.

(1) It suffices to show that $\zeta_{k}$ is hypersimple relative to $\alpha$. This can be done by induction on $k$. $\zeta_{1}$ is hypersimple relative to $\alpha$. The set $\alpha-\zeta_{k}$ is infinite, since it includes the infinite set $\alpha-\zeta_{1}$. Suppose $\zeta_{k^{*}}$ is hypersimple relative to $\alpha$, where $k^{*} \geqq 1$. Assume that $\zeta_{k^{*}+1}$ were not hypersimple relative to $\alpha$. Then there would exist a discrete array of elements in $\alpha$, none of whose rows would be included in $\zeta_{k^{*}+1}$, say $\left\{\delta_{n}\right\}$. Each row of $\left\{\delta_{n}\right\}$ would therefore contain at least one element with a deficiency $\leqq k^{*}$. We claim that $\left\{\delta_{n}\right\}$ must have infinitely 
many rows containing at least one element with deficiency $k^{*}$; for otherwise $\left\{\delta_{n}\right\}$ would have a discrete subarray each row of which would contain at least one element with a deficiency $\leqq k^{*}-1$; this would contradict the induction hypothesis that $\zeta_{k^{*}}$ was hypersimple relative to $\alpha$. Since $\left\{\delta_{n}\right\}$ has infinitely many rows which contain at least one element with deficiency $k^{*}$, we can, by comparing $\left\{\delta_{n}\right\}$ with a recursive enumeration of $\zeta_{k^{*}}$, obtain a subarray of $\left\{\delta_{n}\right\}$ each row of which contains only elements with a deficiency $\geqq k^{*}$ and at least one element with deficiency $k^{*}$. Let $\left\{\rho_{n}\right\}$ be such a subarray of $\left\{\delta_{n}\right\}$. We now transform $\rho_{0}$ into $\sigma_{0}$ in the following manner:

(a) replace every element $x$ of $\rho_{0}$ by the first element in $\{a(n)\}$ which while smaller than $x$ succeeds $x$,

(b) omit all repetitions which might arise because different elements of $\rho_{0}$ are replaced by the same element.

Let $t={ }_{d f} \max \left\{n \mid a(n) \in \sigma_{0}\right\}$. Only finitely many rows of $\left\{\rho_{n}\right\}$ can contain at least one of the elements $a(0), \cdots, a(t)$; thus we can effectively find the first row of $\left\{\rho_{n}\right\}$ all of whose elements succeed $a(t)$ in $\{a(n)\}$, say $\rho_{i}$. We now define $\sigma_{1}$ in terms of $\rho_{i}$ as we defined $\sigma_{0}$ in terms of $\rho_{0}$. Continuing this procedure we transform the discrete array $\left\{\rho_{n}\right\}$ into a discrete array $\left\{\sigma_{n}\right\}$. It now follows from the properties of $\left\{\rho_{n}\right\}$ and the definition of $\left\{\sigma_{n}\right\}$ that every row of $\left\{\sigma_{n}\right\}$ contains at least one element with a deficiency $\leqq k^{*}-1$. Thus $\zeta_{k^{*}}$ would include no row of the discrete array $\left\{\sigma_{n}\right\}$ of elements in $\alpha$; this would contradict the assumption that $\zeta_{k^{*}}$ is hypersimple relative to $\alpha$. We conclude that $\zeta_{k^{*}+1}$ is also hypersimple relative to $\alpha$.

(2) $x \in \beta_{k}$ if and only if $a(x)$ has a deficiency $\geqq k$. We now define $\alpha_{x}^{*}, a_{x, n}^{*}$, and $m(x)$ in the same way as in the proof of the second part of Theorem 1. Let $T_{k, x}$ be the truth table of order $m(x)+1$ such that: if $\alpha_{x}^{*}$ is empty the last column of $T_{k, x}$ is,-- , if $\alpha_{x}^{*}$ is nonempty the last column of $T_{k, x}$ contains the symbol " + " in the $t$ th row if and only if the truth assignment in the $t$ th row contains the symbol " + " at least $k$ times. The decision problem of $\beta_{k}$ is now reducible to that of $\alpha$ by the reduction which associates with every number $x$ the finite sequence $a_{x, 0}^{*}, \cdots, a_{x, m(x)}^{*}$ and the truth table $T_{k, x}$. Thus $\beta_{k} t$-red $\alpha$.

(3) We apply again induction on $k$. We know that $\alpha$ red $\beta_{1}$. Suppose $\alpha$ red $\beta_{k^{*}}$, where $k^{*} \geqq 1$. The fact that $\zeta_{k^{*}+1} \subset \zeta_{k^{*}}$ implies that $\beta_{k^{*}+1} \subset \beta_{k^{*}}$. Suppose $\beta_{k^{*}}-\beta_{k^{*}+1}$ is finite. Then $\alpha$ red $\beta_{k^{*}+1}$ follows from $\alpha$ red $\beta_{k^{*}}$, because $\beta_{k^{*}}$ is many-one reducible to $\beta_{k^{*}+1}$. Now suppose $\beta_{k^{*}}-\beta_{k^{*}+1}$ is infinite, then $\zeta_{k^{*}}-\zeta_{k^{*}+1}$ is also infinite. We can effectively find a 1-1 recursive function $c_{n}$ such that $\zeta_{k^{*}}$ is generated in the sequence $a\left(c_{0}\right), a\left(c_{1}\right), \cdots$. We now raise the following questions about $\zeta_{k^{*}+1}$ : 


$$
\text { “ } a\left(c_{0}\right) \in \zeta_{k^{*}+1} \text { ?", “ } a\left(c_{1}\right) \in \zeta_{k^{*}+1} \text { ?", ... }
$$

Among the correct answers infinitely many must be negative, since $\zeta_{k^{*}}-\zeta_{k^{*}+1}$ is infinite. Let $\left\{d_{n}\right\}$ be the sequence of all $m$ for which there exists an $n$ such that $m=a\left(c_{n}\right) \notin \zeta_{k^{*}+1}$ ordered in the same way as they occur in (I). Observe that for every $n$ the deficiency of $d_{n}$ is exactly $k^{*}$. Let $e_{n}$ be the minimum of the $k^{*}$ elements in $\{a(n)\}$ which while smaller than $d_{n}$ succeed $d_{n}$. Now suppose $x$ is any number. Since there exists an $n_{x}$ such that $\{a(n)\}$ contains only elements $>x$ after $a\left(n_{x}\right)$, there must be a number $t(x)$ such that $e_{t(x)}>x$. But $e_{t(x)}$ is minimal, for the definition of $e_{n}$ implies that $e_{n}$ is minimal for every $n$. Hence $x \in \alpha$ if and only if $x \in\left\{a(0), a(1), \cdots, e_{t(x)}\right\}$. The number $\boldsymbol{e}_{t(x)}$ can be obtained after finitely many questions in (I) are correctly answered. Let $f(x), g(x), h(x)$ be the characteristic functions of $\alpha, \beta_{k^{*}+1}, \zeta_{k^{*}+1}$, respectively. We have proved that $f(x)$ is recursive in $h(a(x))$. But $h(a(x))=g(x)$. It follows that $f(x)$ is recursive in $g(x)$, i.e., that $\alpha \operatorname{red} \beta_{k^{*}+1}$.

CoROllary. Let $a(n)$ be a 1-1 recursive function such that $\rho(a)$ is not recursive. Let $k$ be any number $\geqq 1$. Then:

(1) there are infinitely many elements in $\{a(n)\}$ with a deficiency $\geqq k$,

(2) the set of all elements in $\{a(n)\}$ with a deficiency $<k$ is immune,

(3) the set of all elements in $\{a(n)\}$ with deficiency $k$ is finite or immune.

Proof. The first two parts follow immediately from the fact that $\zeta_{k}$ is hypersimple relative to $\rho(a)$. The third part follows from the second part, because the set of all elements with deficiency $k$ is included in the set of all elements with a deficiency $<k+1$.

NoRTHWESTERN UNIVERSITY 